University of South Florida

DIGITAL COMMONS

Digital Commons @ University of

@ UNIVERSITY OF SOUTH FLORIDA

South Florida

$1-1-2010$

\title{
2010 Annual Report USF Sarasota-Manatee
}

USF

Follow this and additional works at: https://digitalcommons.usf.edu/usf_accountability_reports

\section{Scholar Commons Citation}

USF, "2010 Annual Report USF Sarasota-Manatee" (2010). USF Accountability Reports. 7.

https://digitalcommons.usf.edu/usf_accountability_reports/7

This Article is brought to you for free and open access by the USF Archives at Digital Commons @ University of South Florida. It has been accepted for inclusion in USF Accountability Reports by an authorized administrator of Digital Commons @ University of South Florida. For more information, please contact digitalcommons@usf.edu. 


\section{University of South Florida, Sarasota-Manatee}

Approved by the USF System BOT, December 16th, 2010 


\begin{tabular}{|c|c|c|c|c|c|c|c|}
\hline \multicolumn{8}{|c|}{ University of South Florida 2010 Annual Report } \\
\hline & & & \multicolumn{5}{|c|}{ USF Sarasota-Manatee } \\
\hline Enrollments & \# & $\%$ & \multicolumn{3}{|c|}{ Degree Programs Offered (As of Spr. 10) } & \multicolumn{2}{|c|}{ Carnegie Classification } \\
\hline $\begin{array}{c}\text { TOTAL } \\
\text { (Fall 2009) }\end{array}$ & 1,784 & $100 \%$ & \multicolumn{2}{|l|}{ TOTAL } & 30 & $\begin{array}{c}\text { Undergraduate } \\
\text { Instructional Program: }\end{array}$ & \\
\hline Black & 110 & $6 \%$ & \multicolumn{2}{|c|}{ Baccalaureate } & 23 & Graduate Instructional & \\
\hline Hispanic & 152 & $9 \%$ & \multicolumn{2}{|c|}{ Master's \& Specialist's } & 7 & Program: & \\
\hline White & 1,408 & $79 \%$ & \multicolumn{2}{|c|}{ Research Doctorate } & 0 & Enrollment Profile: & \\
\hline Other & 114 & $6 \%$ & \multicolumn{2}{|c|}{ Professional Doctorate } & 0 & Undergraduate Profile: & $\begin{array}{c}\text { SEPARATE CLASSIFICATION } \\
\text { PENDING }\end{array}$ \\
\hline Full-Time & 693 & $39 \%$ & \multirow{2}{*}{$\begin{array}{c}\text { Faculty } \\
\text { (Fall 2009) }\end{array}$} & \multirow{2}{*}{$\begin{array}{l}\text { Full- } \\
\text { Time }\end{array}$} & \multirow{2}{*}{$\begin{array}{l}\text { Part- } \\
\text { Time }\end{array}$} & Size and Setting: & \\
\hline Part-Time & 1,091 & $61 \%$ & & & & \multirow{2}{*}{ Basic: } & \\
\hline Undergraduate & 1,414 & $79 \%$ & TOTAL & 52 & 5 & & \\
\hline Graduate & 203 & $11 \%$ & Tenure/T. Track & 31 & 1 & \multirow{2}{*}{ Elective Classification: } & \\
\hline Unclassified & 167 & $9 \%$ & Other Faculty/Instr. & 21 & 4 & & \\
\hline
\end{tabular}

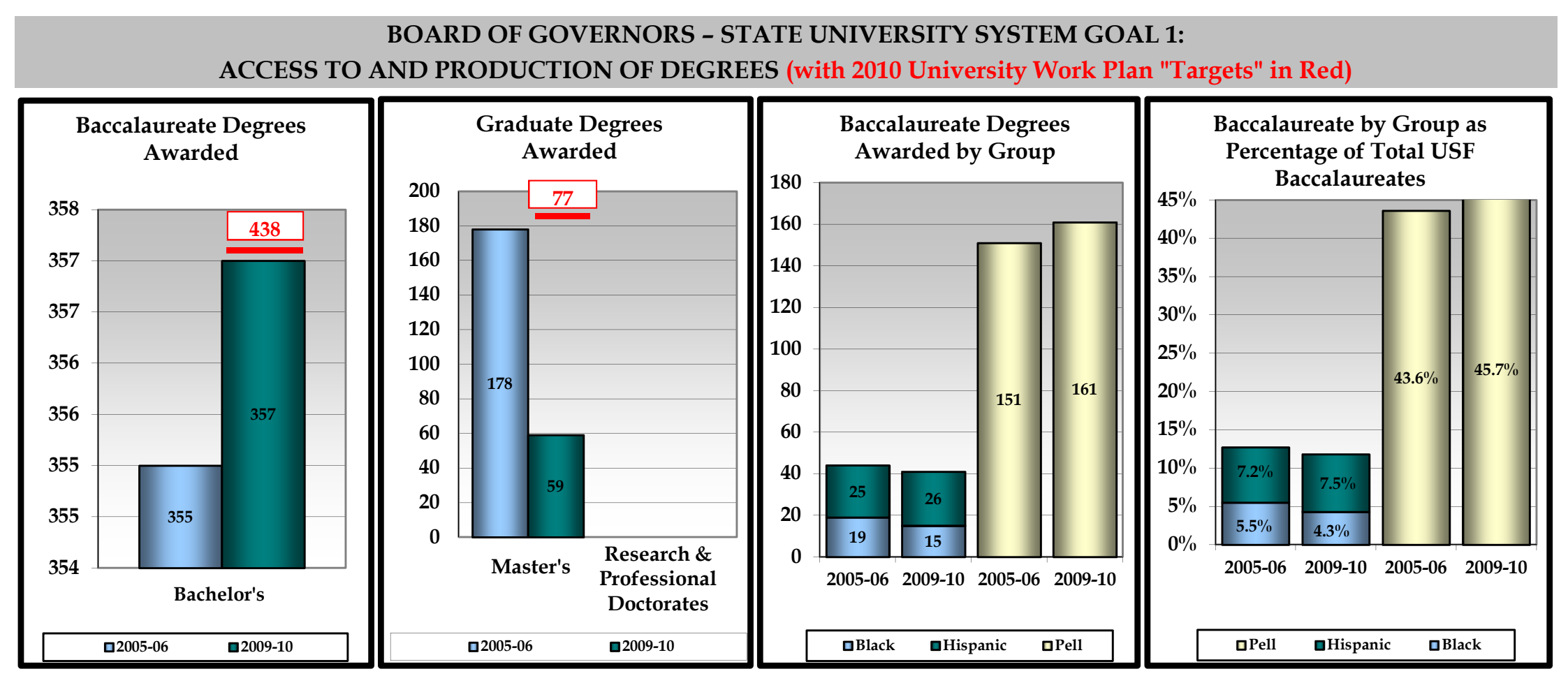

2012 - 2013 Projected Institutional Contributions in RED PRINT. 
BOARD OF GOVERNORS - STATE UNIVERSITY SYSTEM GOAL 2:

MEETING STATEWIDE PROFESSIONAL AND WORKFORCE NEEDS (with 2010 University Work Plan "Targets" in Red)

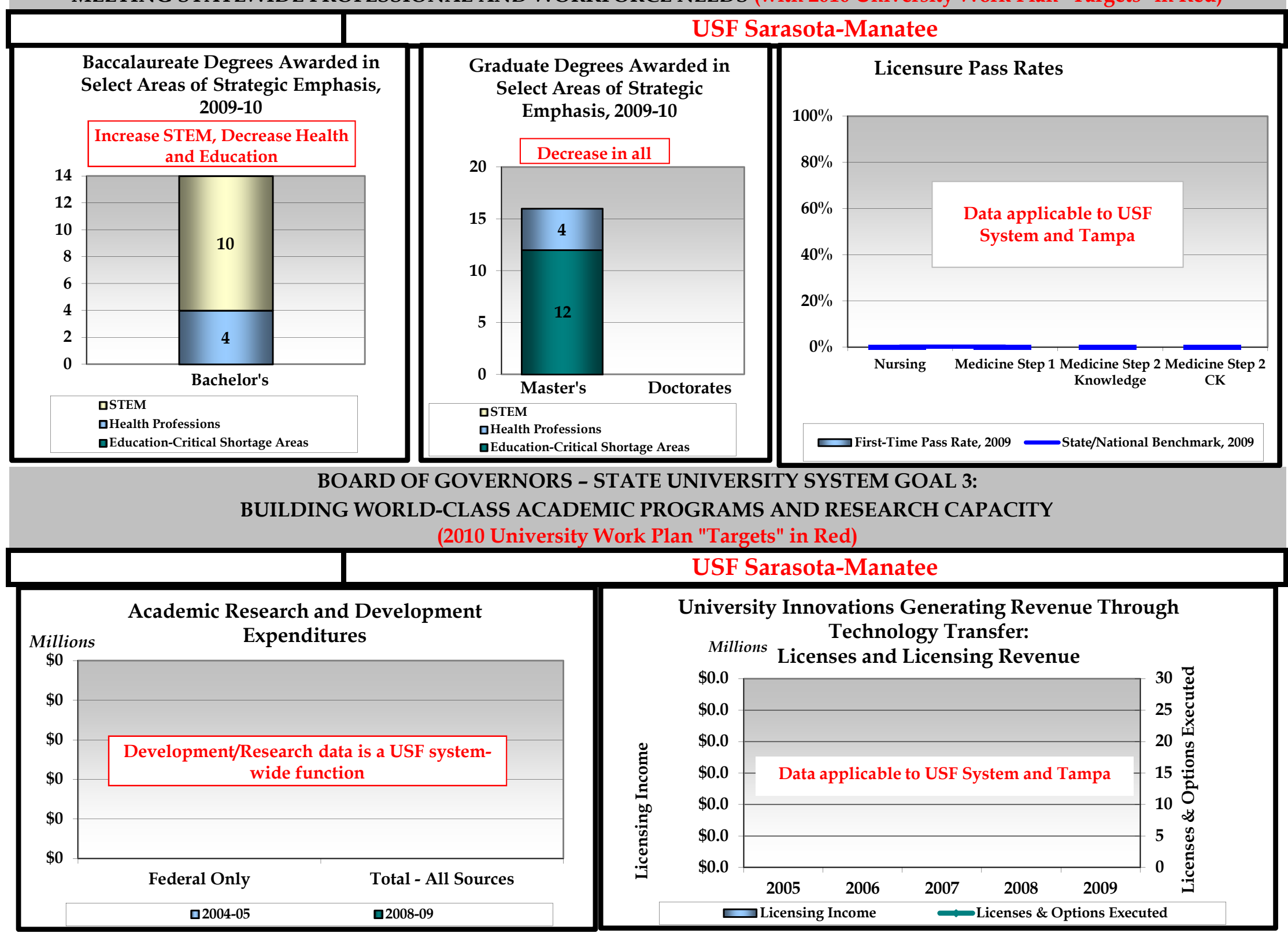

Projected Institutional Contributions in RED PRINT

(2012 - 2013 for TOTAL Degrees in Areas of Strategic Emphasis; 2012 for NCLEX; 2011 -2012 for R\&D, Licences, and Licensing Revenue). 


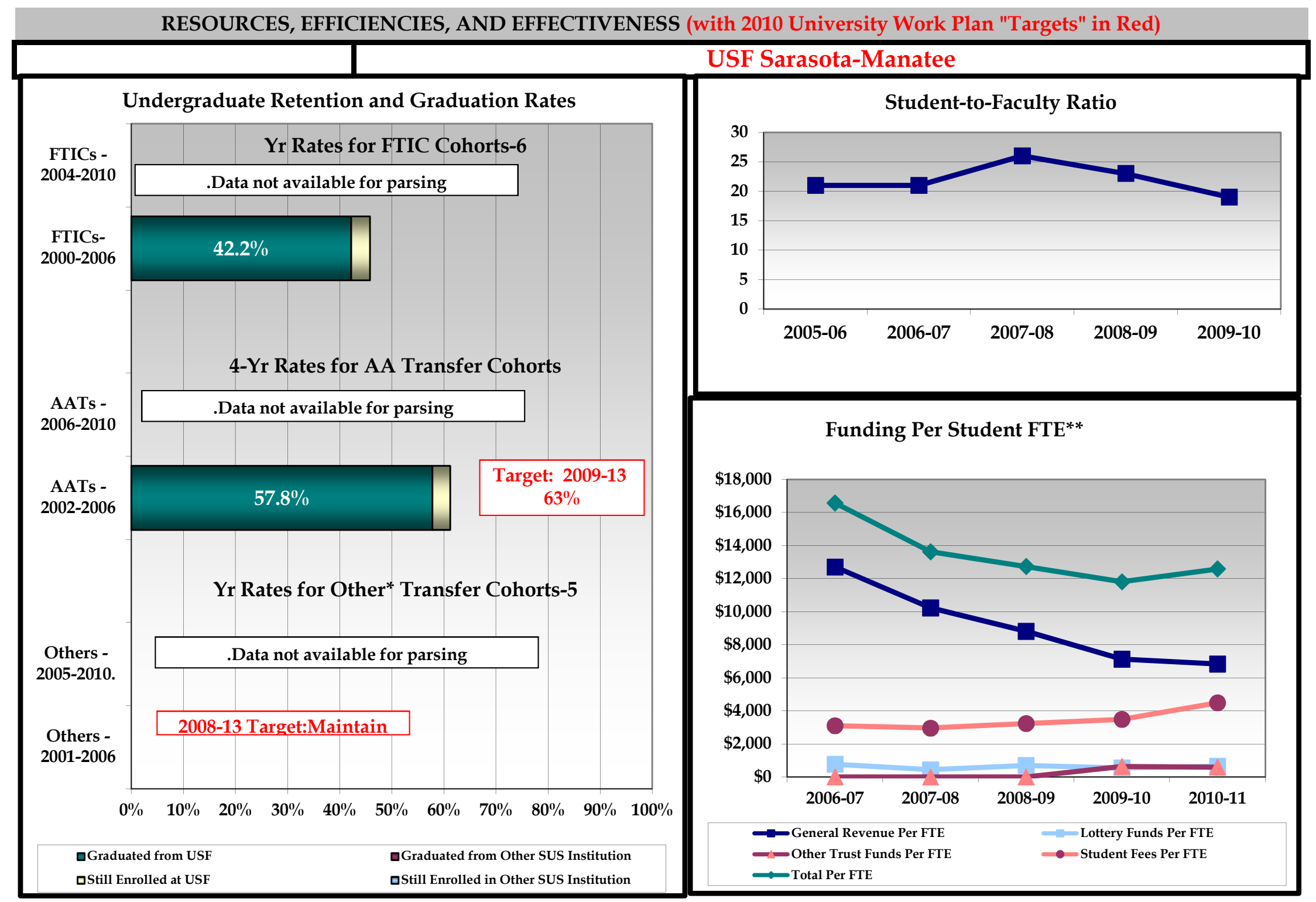

* The composition of "Other Transfer" cohorts may vary greatly by institution and by year.

** FTE for this metric uses the standard IPEDS definition of FTE, equal to 30 credit hours for undergraduates and 24 for graduates.

Graduation Rate from SAME Institution - Projected Institutional Contributions in RED PRINT. 


\section{Key University Achievements}

Note: Limit to one-page. Enter a maximum of three issues per category. Only use 11-point Book Antiqua font.

\section{Student awards/achievements}

1. 2010 Florida Achievement Award presented to a USFSM College of Education senior student for her outstanding contributions and services to the community.

\section{- Faculty awards/achievements}

1. USFSM professor Dr. J. Unnever given the Nationally Prestigious Donal A. J. MacNamara Award for outstanding publication from Academy of Criminal Justice Sciences.

\section{- Program awards/achievements}

1. School of Hotel and Restaurant Management is working to build $3+1$ programs with 6 (six) different international universities.

2. USFSM College of Education sponsored the 2010 Children's Literature Symposium - a joint conference with West Central Region F.A.T.E.

\section{- Research awards/achievements}

1. A College of Arts \& Sciences professor received a $\$ 131,566$ sub-contract from SCOPE to conduct research for The Aging with Dignity and Independence Initiative.

2. The article published by two College of Education professors in the Journal of Intergenerational Relationships (JIR): “Lessons from Erikson: A look at autonomy across lifespan" has been cited more than any other in the journal. (202 times)

3. A College of Education professor was awarded as a Co-PI a grant from the Social Science Research and Humanities Council of Canada to research children affected by war in northern Uganda. She will be traveling to Uganda in November.

\section{- Institutional awards/achievements}

1. Fall 2010, USFSM opened its new USFSM @ North Port instructional site. The facility allows USFSM to provide access to baccalaureate and master's programs to students living in south Sarasota County.

2. USF Sarasota-Manatee received a one-year, $\$ 247,500$ grant from the Health Resources and Services Administration (HRSA). Outcomes include a five-year strategic plan for delivery of health care degrees/certificates that meet workforce needs in Sarasota and Manatee Counties.

3. USF Sarasota-Manatee's College of Business received a two-year \$170,000 grant from the Gulf Coast Community Foundation of Venice to improve the quality and quantity of business courses to residents of south Sarasota County. 


\section{INTRODUCTION}

University of South Florida, Sarasota-Manatee

\section{Mission}

The University of South Florida Sarasota-Manatee delivers quality education to upper-division baccalaureate and master's students in an active research environment. We support students in attaining their highest potential by nurturing leadership, critical thinking, and an appreciation for learning. We advance cultural, social, environmental, and economic well-being for all we serve.

\section{Vision}

\section{HOMETOWN CAMPUS, GLOBAL IMPACT}

The University of South Florida Sarasota-Manatee aspires to be a leader in higher education, delivering accessible academic programs and contributing significant research, while inspiring continuous improvement locally and globally.

\section{Other Contextual Introductory Comments}

In October 2010, USF Sarasota-Manatee submitted its Certification of Compliance for separate accreditation to the Southern Association of Colleges and Schools (SACS). The campus will be site visited by the accreditor in November 2010. With anticipated accreditation separate from the Tampa Campus in June 2011.

\section{BOARD OF GOVERNORS - STATE UNIVERSITY SYSTEM GOAL 1: ACCESS TO AND PRODUCTION OF DEGREES}

USF Sarasota-Manatee Florida Community College transfers take an average of 2.23 years to graduate despite the fact that $65 \%$ of students are working at least part-time. USF SarasotaManatee also has a consistently high retention rate of over $72 \%$ each cohort year. The success of the campus' students in completing degrees can be attributed to the following: 1) small class sizes; 2) expanded daytime, evening, online, and blended course offerings; 3 ) advising efforts such as the Still To Register Campaign in which advisors track and follow-up with students to ensure their timely registration each semester; and 4) diligent course tracking and scheduling to ensure the courses that students need are offered each semester.

Until 2009-10, undergraduate degree production grew each year after the campus moved to its own site in 2006 . The decline in 2009-10 in both graduate and undergraduate degrees resulted when USF Sarasota-Manatee recoded to Tampa any students in Tampa-only degree programs, in anticipation of separate SACS accreditation. When in the past, these students would have graduated from USF SarasotaManatee; instead they were counted in the USF Tampa figures.

In the future, when USF Sarasota-Manatee is awarded separate accreditation, the campus hopes to increase the number of degree programs it offers at both the undergraduate and graduate levels. Currently, the campus offers fifteen (15) baccalaureate degree programs and six (6) master's degree programs.

Approved by the USF System BOT, December 16th, 2010 


\section{BOARD OF GOVERNORS - STATE UNIVERSITY SYSTEM GOAL 2: MEETING STATEWIDE PROFESSIONAL AND WORKFORCE NEEDS}

USF Sarasota-Manatee has made tremendous strides in the past few years to further meet the needs of its community and the State of Florida. All twenty-one (21) degree programs offered at the campus are closely aligned with workforce needs of the area. For example our business degree programs help to educate those seeking employment in the ever-growing fields of accountancy, management, sales, and marketing. USF Sarasota-Manatee's College of Arts \& Sciences produces graduates ready for work in any number of fields with their strong programs in English, Psychology, Criminology, History, and Information Technology. USF Sarasota-Manatee's own School of Hotel \& Restaurant Management, closely aligned with the local tourism industry, is growing in bachelor's degree enrollments each year. The School is currently going through the approval process for a master's degree. Finally, the USF Sarasota-Manatee's College of Education, produces not simply teachers, but a workforce of highly qualified educators.

\section{BOARD OF GOVERNORS - STATE UNIVERSITY SYSTEM GOAL 3: BUILDING WORLD-CLASS ACADEMIC PROGRAMS AND RESEARCH CAPACITY}

USF Sarasota-Manatee faculty members are committed to conducting relevant research that will contribute to the public good. During the academic year 2009-10, USF SarasotaManatee faculty members applied for more than $\$ 3.34$ million dollars in grants and devoted between $5 \%$ and $50 \%$ of their workloads to research efforts. The following are but a few highlights of their efforts:
- A USF Sarasota-Manatee professor given the Nationally Prestigious Donal A. J. MacNamara Award for outstanding publication from Academy of Criminal Justice Sciences

- USFSM College of Education sponsored the 2010 Children's Literature Symposium - a joint conference with West Central Region F.A.T.E. with the theme of Engaging a New Generation of Readers: The Value of Evolution of Story. The symposium brought presentations from educators, scholars, actors, education consultants, critics, librarians and graduate students to address shifts and developments in literature and explore emerging genres.

- The article published by two College of Education professors in the Journal of Intergenerational Relationships (JIR): “Lessons from Erikson: A look at autonomy across lifespan" has been cited more than any other in the journal. (202 times)

\section{BOARD OF GOVERNORS - STATE UNIVERSITY SYSTEM GOAL 4: MEETING COMMUNITY NEEDS AND FULFILLING UNIQUE INSTITUTIONAL RESPONSIBILITIES}

USF Sarasota-Manatee is committed to serving the needs of Sarasota, Manatee, and DeSoto counties. Part of that commitment is the campus' mission to advance the "economic well-being" of our surrounding communities. The following recent efforts evidence this commitment:

Approved by the USF System BOT, December 16th, 2010 
- Fall 2010 marked the grand opening of the new USF Sarasota-Manatee @ North Port instructional site. The site, originally housed on the State College of Florida's Venice campus, moved into the newly renovated building to accommodate the ever steady increase of south county student enrollments. The site offers coursework towards degrees in criminology, psychology, interdisciplinary social sciences, general business administration, elementary education, and educational leadership.

- USF Sarasota-Manatee's College of Business received a two-year $\$ 170,000$ grant from the Gulf Coast Community Foundation of Venice to improve the quality and quantity of business courses to residents of south Sarasota County. The funds are being used to finance a business advisor/recruiter and faculty in management and marketing who are delivering course work at the North Port instructional site of USF Sarasota-Manatee.

- USF Sarasota-Manatee received a one-year, $\$ 247,500$ grant from the Health Resources and Services Administration (HRSA) entitled, "A Blueprint for Health Professions Education at USF SarasotaManatee." Outcomes include a five-year strategic plan for delivery of health care degrees/certificates that meet workforce needs in Sarasota and Manatee Counties.

- USF Sarasota-Manatee received a \$131,566 sub-contract from Sarasota County Openly Plans for Excellence (SCOPE) to conduct research for The Aging with Dignity and Independence Initiative, a multi-year project with an end goal of creating innovative and technological approaches to help people 65 years and older age with dignity and independence in the community. The research will engage residents through community forums, focus groups and surveys, seeking to learn from the experiences of older adults as well as the broader community. The Aging with Dignity and Independence Initiative is funded by The Patterson Foundation.

- Twenty-two local professionals received a professional development certificate in Non-profit Management from USF Sarasota-Manatee this past spring. The program, developed by USFSM and The Community Foundation of Sarasota County, was a ten week course for potential and current nonprofit managers and leaders to prepare emerging professionals for the unique challenges in today's nonprofit climate.

- USFSM's annual Brunch on the Bay has raised over \$2.7 million dollars since 1994 to support endowed and First Generation student scholarships.

\section{PROGRESS ON PRIMARY INSTITUTIONAL GOALS AND METRICS AS OUTLINED IN THE UNIVERSITY WORK PLAN}

\section{USF Sarasota-Manatee Strategic Goals}

1. Learner-Centered Environment: Be a diverse, collaborative, and intellectually challenging campus with high quality academic programs and support services for students, faculty, staff, and community.

With strategic focus on teaching and learning, research, programming, campus climate, and community USF Sarasota-Manatee has met and seeks to exceed its objectives for this goal.

Approved by the USF System BOT, December 16th, 2010 
- Presently, 75\% of USF Sarasota-Manatee student credit hours (SCH) are taught by full-time resident faculty. The campus will maintain this percentage with a goal of even higher ratios once funding for additional faculty members becomes available.

- Likewise, 75\% of USF Sarasota-Manatee faculty members teaching undergraduate students have the doctorate degree in the discipline in which they are teaching.

2. Technology: Provide and encourage strategic use of electronic learning technology.

Student convenience and teaching and learning enhancement foci have resulted in a 9\% increase in the number of distance learning courses in 2009-10 over 200809 levels.

USF Sarasota-Manatee continues to focus curricular efforts on using technology to develop convenient schedules for student learning, such as online courses, distance learning, and hybrid courses.

3. Program Growth: Promote expansion of distinctive upper-division baccalaureate, master's, and research initiatives that serve local and global needs.

Through the efforts of seeking separate accreditation, USF Sarasota-Manatee has worked to ensure degree programs are complete and that students have access to resident faculty and a full course schedule to ensure timely degree obtainment.
USF Sarasota-Manatee plans to increase the number of programs it offers the community in the near future. In concert with the release of the 2010 census data, USF Sarasota-Manatee will conduct a comprehensive environmental scan of the three-county area to identify educational needs and growing markets.

4. Professional and Continuing Education: Provide advanced professional and continuing education that serves community needs.

USF Sarasota-Manatee continued its efforts to meet workforce and professional development needs in Sarasota, Manatee, and DeSoto counties. In addition to its academic programs, USFSM has also strategically provided continuing education credits through its College of Business and the Institute for Public Policy and Leadership.

5. Financial Stability: Grow and diversify the resource base.

USF Sarasota-Manatee lost \$5.96 million in recurring funding from the State of Florida over the last three years. USF Sarasota-Manatee will be seeking \$2 million each year for the next three years from the State Legislature to support the separate accreditation process and to grow into a four-year degree institution. Funds will be used to hire additional faculty to teach general education courses and pre-requisite courses in business and education. 
6. Assessment: Expand the system of self-study and continuous improvement.

USF Sarasota-Manatee is committed to engaging in selfassessment in both its academic programs and administrative units. 2009-10 marked the third cycle of academic assessment within the newly autonomous USFSM Colleges.

7. Accreditation: Work toward institutional autonomy within the USF system with separate regional professional accreditations.

In September 2010, USF Sarasota-Manatee submitted its Certification of Compliance to the Southern Association of Colleges and Schools. The campus will be site visited by the accreditors in November 2010 with anticipated accreditation separate from the Tampa Campus in June 2011.

\section{ADDITIONAL INFORMATION ON QUALITY, RESOURCES, EFFICIENCIES, AND EFFECTIVENESS}

Since 2006-07, USFSM has reduced its electrical KWH demand by $21 \%$.

\section{ADDITIONAL RESOURCES}

University Strategic Plan

http://www.sarasota.usf.edu/ir/Strategic_Plan/StrategicPla nHome.php

\section{College Navigator}

http://nces.ed.gov/collegenavigator/?q=South+Florida\&s=al

$1 \&$ id $=451671$ \#fedloans

University Institutional Research Unit

http://www.sarasota.usf.edu/ir/ 


\section{Section 1 - Financial Resources (including Health-Science Center \& IFAS, if applicable)}

Table 1A. Education and General Revenues

Table 1B. Education and General Expenditures

Table 1C. Funding per Student

Table 1D. Other Budget Entities

Table 1E. Total Revenues and Expenditures

Table 1F. Voluntary Support of Higher Education

Table 1G. Federal Stimulus Dollars (ARRA)

\section{Section 2 - Personnel}

Table 2A. Personnel Headcount

\section{Section 3 - Enrollment \& Space}

Table 3A. University Full-time Enrollment (FTE)

Table 3B. Enrollment by Location

Table 3C. Space Utilization

\section{Section 4 - Undergraduate Education}

Table 4A. Baccalaureate Degree Program Changes in AY 2009-2010

Table 4B. $\quad$ First-Year Persistence Rates

Table 4C. Federal Definition - Undergraduate Progression and Graduation Rates

for Full-time First-time-in-College (FTIC) Students

Table 4D. SUS - Undergraduate Progression and Graduation Rates

for First-time-in-College (FTIC) Students

Table 4E. SUS - Undergraduate Progression and Graduation Rates for AA Transfer Students

Table 4F. $\quad$ SUS - Undergraduate Progression and Graduation Rates

$$
\text { for Other Transfer Students }
$$

Table 4G. Baccalaureate Degrees Awarded

Table 4H. Baccalaureate Degrees Awarded in Areas of Strategic Emphasis

Table 4I. Baccalaureate Degrees Awarded to Underrepresented Groups

Table 4J. Baccalaureate Completion Without Excess Credit Hours

Table 4K. Undergraduate Course Offerings

Table 4L. Faculty Teaching Undergraduates

Table 4M. Undergraduate Instructional Faculty Compensation

Table 4 N. Student/Faculty Ratio

Table 4O. Licensure Pass Rates

Table 4P. Tuition Differential Fee

\section{Section 5 - Graduate Education}

Table 5A. Graduate Degree Programs Changes in AY 2009-2010

Table 5B. Graduate Degrees Awarded

Table 5C. Graduate Degrees Awarded in Areas of Strategic Emphasis

Table 5D. Licensure Pass Rates

\section{Section 6 - Research and Economic Development}

Table 6A. Research and Development

Table 6B. Centers of Excellence

Table 6C. State University Research Commercialization Assistance Grants

Table 6D. 21st Century World Class Scholars Program

\section{Appendix I - Definitions}




\begin{tabular}{|c|c|c|c|c|c|}
\hline \multicolumn{6}{|c|}{$\begin{array}{c}\text { STATE UNIVERSITY SYSTEM - 2010 ANNUAL REPORT } \\
\text { Section 1 - Financial Resources }\end{array}$} \\
\hline \multicolumn{6}{|c|}{ University of South Florida-Sarasota-Manatee } \\
\hline \multicolumn{6}{|c|}{ Table 1A. University Education and General Revenues } \\
\hline $\begin{array}{c}\text { Recurring State Funds } \\
\text { (GR \& Lottery) }\end{array}$ & $\$ 16,344,059$ & $\$ 14,941,901$ & $\$ 13,959,366$ & $\$ 11,996,133$ & $\$ 12,042,638$ \\
\hline $\begin{array}{c}\text { Non-Recurring State Funds } \\
\text { (GR \& Lottery) }\end{array}$ & $\$ 0$ & $\$ 150,120$ & $\$ 146,382$ & $\$ 85,400$ & $\$ 93,391$ \\
\hline $\begin{array}{c}\text { Tuition } \\
\text { (Resident/ Non-Resident) }\end{array}$ & $\$ 3,640,032$ & $\$ 4,184,045$ & $\$ 4,782,090$ & $\$ 5,234,584$ & $\$ 6,800,800$ \\
\hline $\begin{array}{c}\text { Tuition Differential Fee } \\
\text { Actual }\end{array}$ & $\$ 0$ & $\$ 0$ & $\$ 0$ & $\$ 193,210$ & $\$ 429,618$ \\
\hline $\begin{array}{c}\text { Other Revenues } \\
\text { (Includes Misc. Fees \& Fines) }\end{array}$ & $\$ 112,837$ & $\$ 7,722$ & $\$ 8,803$ & $\$ 66,227$ & $\$ 59,800$ \\
\hline $\begin{array}{c}\text { Phosphate Research } \\
\text { Trust Fund }\end{array}$ & $\$ 0$ & $\$ 0$ & $\$ 0$ & $\$ 0$ & $\$ 0$ \\
\hline $\begin{array}{c}\text { Federal Stimulus Funds } \\
\text { TOTAL }\end{array}$ & $\$ 20,096,928$ & $\$ 19,283,788$ & $\$ 18,896,641$ & $\$ 18,587,680$ & $\$ 20,394,703$ \\
\hline
\end{tabular}

*Note: 2010-11 estimates for the USF System report are accepted at the request of the BOG. It should be noted however, that a discrepancy in the amount of transfers between the main campus and HSC is reflected in this table and in the HSC corresponding table. In turn, the individual campus reports will not roll up to these reflected totals and will corrections will be reflected in the 2011 Annual Report.

\begin{tabular}{|c|c|c|c|c|c|}
\hline & $\begin{array}{r}2006-07 \\
\text { Actual }\end{array}$ & $\begin{array}{c}\text { 2007-08 } \\
\text { Actual }\end{array}$ & $\begin{array}{r}\text { 2008-09 } \\
\text { Actual }\end{array}$ & $\begin{array}{c}\text { 2009-10 } \\
\text { Actual }\end{array}$ & $\begin{array}{c}\text { 2010-11 } \\
\text { Estimates }\end{array}$ \\
\hline Instruction/Research & $\$ 9,952,983$ & $\$ 10,457,953$ & $\$ 9,423,525$ & $\$ 9,786,291$ & $\$ 13,185,794$ \\
\hline Institutes and Research Centers & $\$ 0$ & $\$ 0$ & $\$ 0$ & $\$ 0$ & $\$ 0$ \\
\hline PO\&M & $\$ 1,312,669$ & $\$ 1,268,101$ & $\$ 837,867$ & $\$ 920,903$ & $\$ 1,333,216$ \\
\hline $\begin{array}{c}\text { Administration } \\
\text { and Support Services }\end{array}$ & $\$ 3,374,878$ & $\$ 2,282,003$ & $\$ 2,154,447$ & $\$ 2,528,316$ & $\$ 3,126,499$ \\
\hline Radio/TV & $\$ 0$ & $\$ 0$ & $\$ 0$ & $\$ 0$ & $\$ 0$ \\
\hline Library/Audio Visual & $\$ 802,613$ & $\$ 707,931$ & $\$ 545,797$ & $\$ 1,191,766$ & $\$ 701,395$ \\
\hline Museums and Galleries & $\$ 0$ & $\$ 0$ & $\$ 0$ & $\$ 0$ & $\$ 0$ \\
\hline Agricultural Extension & $\$ 0$ & $\$ 0$ & $\$ 0$ & $\$ 0$ & $\$ 0$ \\
\hline Allied Clinics & $\$ 0$ & $\$ 0$ & $\$ 0$ & $\$ 0$ & $\$ 0$ \\
\hline Student Services & $\$ 1,184,306$ & $\$ 2,493,848$ & $\$ 1,829,878$ & $\$ 1,829,151$ & $\$ 2,039,577$ \\
\hline Intercollegiate Athletics & $\$ 0$ & $\$ 0$ & $\$ 0$ & $\$ 0$ & $\$ 0$ \\
\hline TOTAL & $\$ 16,627,448$ & $\$ 17,209,836$ & $\$ 14,791,514$ & $\$ 16,256,427$ & $\$ 20,386,481$ \\
\hline
\end{tabular}


The table reports the actual and estimated amount of expenditures from revenues appropriated by the legislature for each

fiscal year. The expenditures are classified by Program Component (i.e., Instruction/ Research, PO\&M, Administration, etc...) for activities directly related to instruction, research and public service. The table does not include expenditures classified as non-operating expenditures (i.e., to service asset-related debts), and therefore excludes a small portion of the amount

appropriated each year by the legislature. Also, the table does not include expenditures from funds carried forward from previous years. 


\begin{tabular}{|c|c|c|c|c|c|}
\hline \multicolumn{6}{|c|}{$\begin{array}{l}\text { STATE UNIVERSITY SYSTEM - } 2010 \text { ANNUAL REPORT } \\
\text { Section } 1 \text { - Financial Resources }\end{array}$} \\
\hline \multicolumn{6}{|c|}{ University of South Florida--Sarasota-Manatee } \\
\hline \multicolumn{6}{|c|}{ Table 1C. Funding per Full-Time Equivalent (FTE) Student } \\
\hline & 2006-07 & 2007-08 & 2008-09 & 2009-10 & 2010-11 \\
\hline \multicolumn{6}{|l|}{ Appropriated Funding per FTE } \\
\hline General Revenue per FTE & $\$ 12,712$ & $\$ 10,227$ & $\$ 8,813$ & $\$ 7,130$ & $\$ 6,842$ \\
\hline Lottery Funds per FTE & $\$ 773$ & $\$ 438$ & $\$ 693$ & $\$ 546$ & $\$ 650$ \\
\hline $\begin{array}{l}\text { Tuition \& Fees per FTE } \\
\text { (based on Budget Authority) }\end{array}$ & $\$ 3,096$ & $\$ 2,962$ & $\$ 3,228$ & $\$ 3,490$ & $\$ 4,495$ \\
\hline Other Trust Funds per FTE & $\$ 0$ & $\$ 0$ & $\$ 0$ & $\$ 643$ & $\$ 598$ \\
\hline Total per FTE & $\$ 16,581$ & $\$ 13,627$ & $\$ 12,734$ & $\$ 11,809$ & $\$ 12,585$ \\
\hline Actual Funding per FTE & 2006-07 & $2007-08$ & 2008-09 & 2009-10 & est. $2010-11$ \\
\hline $\begin{array}{l}\text { Tuition \& Fees per FTE } \\
\text { (based on Actual Collections) }\end{array}$ & $\$ 3,096$ & $\$ 2,962$ & $\$ 3,228$ & $\$ 3,490$ & $\$ 4,500$ \\
\hline Actual Total per FTE & $\$ 16,581$ & $\$ 13,627$ & $\$ 12,734$ & $\$ 11,809$ & $\$ 12,590$ \\
\hline \multicolumn{6}{|c|}{$\begin{array}{l}\text { *Note: Change to reporting of these data on funding per student FTE may result in differences in years prior to } 2009-10 \text { as } \\
\text { history was not modified to reflect the new reporting methodology. }\end{array}$} \\
\hline \multicolumn{6}{|c|}{$\begin{array}{l}* \text { Note: Figures are approved for the } 2010 \text { Annual Report but it should be noted however,discrepancies due to transfers not } \\
\text { reflected in this report may result; figures will be corrected in the following annual report. }\end{array}$} \\
\hline \multicolumn{6}{|c|}{$\begin{array}{l}\text { Notes: (1) FTE is based on actual FTE, not funded FTE; (2) Does not include Health-Science Center funds or FTE; (3) } \\
\text { FTE for these metrics uses the standard IPEDS definition of FTE, equal to } 30 \text { credit hours for undergraduates and } 24 \\
\text { for graduates; and (4) Actual funding per student is based on actual tuition and E\&G fees (does not include local } \\
\text { fees) collected. }\end{array}$} \\
\hline \multicolumn{6}{|c|}{ Table 1D. University Other Budget Entities } \\
\hline & $\begin{array}{r}2006-07 \\
\text { Actual } \\
\end{array}$ & $\begin{array}{r}2007-08 \\
\text { Actual } \\
\end{array}$ & $\begin{array}{r}2008-09 \\
\text { Actual } \\
\end{array}$ & $\begin{array}{r}2009-10 \\
\text { Actual } \\
\end{array}$ & $\begin{array}{c}2010-11 \\
\text { Estimates }\end{array}$ \\
\hline \multicolumn{6}{|l|}{ Contracts \& Grants } \\
\hline Revenues & \multirow{2}{*}{\multicolumn{5}{|c|}{ Contracts \& Grants data is a USF system-wide function and consolidated system data is only available at this time. }} \\
\hline Expenditures & & & & & \\
\hline \multicolumn{6}{|l|}{ Auxiliary Enterprises } \\
\hline Revenues & \multirow{2}{*}{\multicolumn{5}{|c|}{ Contracts \& Grants data is a USF system-wide function and consolidated system data is only available at this time }} \\
\hline Expenditures & & & & & \\
\hline \multicolumn{6}{|l|}{ Local Funds } \\
\hline Revenues & \multirow{2}{*}{\multicolumn{5}{|c|}{ Contracts \& Grants data is a USF system-wide function and consolidated system data is only available at this time. }} \\
\hline Expenditures & & & & & \\
\hline \multicolumn{6}{|c|}{ Table 1E. University's Total Revenues and Expenditures } \\
\hline & $\begin{array}{r}2006-07 \\
\text { Actual }\end{array}$ & $\begin{array}{r}2007-08 \\
\text { Actual } \\
\end{array}$ & $\begin{array}{r}2008-09 \\
\text { Actual } \\
\end{array}$ & $\begin{array}{r}\text { 2009-10 } \\
\text { Actual } \\
\end{array}$ & $\begin{array}{c}2010-11 \\
\text { Estimates }\end{array}$ \\
\hline Revenues & $\$ 20,096,928$ & $\$ 19,283,788$ & $\$ 18,896,641$ & $\$ 18,587,680$ & $\$ 20,394,703$ \\
\hline Expenditures & $\$ 16,627,448$ & $\$ 17,209,836$ & $\$ 14,791,514$ & $\$ 16,256,427$ & $\$ 20,386,481$ \\
\hline
\end{tabular}


*Note: 2010-11 estimates for the USF System report are accepted at the request of the BOG. It should be noted however, that a discrepancy in the amount of transfers between the main campus and HSC is reflected in this table and in the HSC corresponding table. In turn, the individual campus reports will not roll up to these reflected totals and will corrections will be reflected in the 2011 Annual Report. 


\begin{tabular}{|c|c|c|c|c|c|}
\hline \multicolumn{6}{|c|}{$\begin{array}{l}\text { STATE UNIVERSITY SYSTEM - } 2010 \text { ANNUAL REPORT } \\
\text { Section } 1 \text { - Financial Resources }\end{array}$} \\
\hline \multicolumn{6}{|c|}{ University of South Florida--Sarasota-Manatee } \\
\hline \multicolumn{6}{|c|}{ Table 1F. Voluntary Support of Higher Education } \\
\hline & 2004-2005 & $2005-2006$ & 2006-2007 & $2007-2008$ & 2008-2009 \\
\hline $\begin{array}{c}\text { Endowment Market Value } \\
\text { (Thousand \$) }\end{array}$ & \multirow{3}{*}{\multicolumn{5}{|c|}{$\begin{array}{c}\text { Development/Research data is a USF system-wide function. Consolidated } \\
\text { system data is only available at this time. }\end{array}$}} \\
\hline $\begin{array}{l}\text { Annual Gifts Received } \\
(\$)\end{array}$ & & & & & \\
\hline $\begin{array}{l}\text { Percentage of Graduates Who Are } \\
\text { Alumni Donors }\end{array}$ & & & & & \\
\hline
\end{tabular}

\begin{tabular}{|l|c|c|}
\hline Table 1G. University Federal Stimulus Dollars (ARRA) \\
\hline Proposed Operating Budget Detail & Actual 2009-10 & Proposed 2010-11 \\
\hline Jobs Saved/Created & & $\$ 968,456$ \\
\hline Scholarships & $\$ 1,012,126$ & $\$ 0$ \\
\hline Library Resources & $\$ 0$ & $\$ 0$ \\
\hline Building Repairs/Alterations & $\$ 0$ & $\$ 0$ \\
\hline Motor Vehicles & $\$ 0$ & $\$ 0$ \\
\hline Printing & $\$ 0$ & $\$ 0$ \\
\hline Furniture \& Equipment & $\$ 0$ & $\$ 0$ \\
\hline Information Technology Equipment & $\$ 0$ & $\$ 0$ \\
\hline Financial Aid to Medical Students & $\$ 0$ & $\$ 0$ \\
\hline Other: & $\$ 0$ & $\$ 0$ \\
\hline TOTAL & $\$ 0$ & $\$ 968,456$ \\
\hline
\end{tabular}


STATE UNIVERSITY SYSTEM - 2010 ANNUAL REPORT

Section 2 - Personnel

\section{University of South Florida--Sarasota-Manatee}

\section{Table 2A. Personnel Headcount}

\begin{tabular}{|c|c|c|c|c|c|c|c|c|c|c|}
\hline & \multicolumn{2}{|c|}{ Fall 2005} & \multicolumn{2}{|c|}{ Fall 2006} & \multicolumn{2}{|c|}{ Fall 2007} & \multicolumn{2}{|c|}{ Fall 2008} & \multicolumn{2}{|c|}{ Fall 2009} \\
\hline & FT & PT & FT & PT & FT & PT & FT & PT & FT & PT \\
\hline $\begin{array}{c}\text { Faculty } \\
\text { Tenure/ Tenure-track }\end{array}$ & 30 & 3 & 26 & 2 & 25 & 1 & 28 & 1 & 31 & 1 \\
\hline $\begin{array}{c}\text { Faculty } \\
\text { Non-Tenure Track }\end{array}$ & 11 & 11 & 16 & 10 & 14 & 11 & 22 & 8 & 21 & 4 \\
\hline $\begin{array}{l}\text { Instructors Without } \\
\text { Faculty Status }\end{array}$ & 0 & 0 & 0 & 0 & 0 & 0 & 0 & 0 & 0 & 0 \\
\hline $\begin{array}{c}\text { Graduate Assistants/ } \\
\text { Associates }\end{array}$ & & 5 & & 8 & & 8 & & 5 & & 2 \\
\hline $\begin{array}{c}\text { Executive/ } \\
\text { Administrative/ Managerial }\end{array}$ & 13 & 0 & 15 & 0 & 25 & 0 & 23 & 0 & 27 & 0 \\
\hline Other Professional & 37 & 1 & 40 & 2 & 33 & 2 & 38 & 2 & 34 & 1 \\
\hline Non-Professional & 22 & 0 & 33 & 0 & 45 & 0 & 38 & 0 & 38 & 0 \\
\hline TOTAL PERSONNEL & & & & & & & & & & \\
\hline
\end{tabular}




\begin{tabular}{|c|c|c|c|c|c|c|}
\hline \multicolumn{7}{|c|}{$\begin{array}{l}\text { STATE UNIVERSITY SYSTEM - } 2010 \text { ANNUAL REPORT } \\
\text { Section } 3 \text { - Enrollment \& Space }\end{array}$} \\
\hline \multicolumn{7}{|c|}{ University of South Florida--Sarasota-Manatee } \\
\hline \multicolumn{7}{|c|}{ Table 3A. University Full-Time Enrollment (FTE) } \\
\hline & \multicolumn{2}{|c|}{ 2008-09 } & \multicolumn{2}{|c|}{ 2009-10 } & \multicolumn{2}{|c|}{ 2010-11 } \\
\hline & Funded & Actual & Funded & Actual & Funded & Estimated \\
\hline \multicolumn{7}{|l|}{ Florida Residents } \\
\hline Lower & 0 & 7 & 0 & 32 & 0 & 0 \\
\hline Upper & 798 & 905 & 798 & 966 & 798 & 1,064 \\
\hline Grad I & 182 & 180 & 182 & 161 & 182 & 132 \\
\hline Grad II & 0 & 2 & 0 & 0 & 0 & 0 \\
\hline Total & 980 & 1,094 & 980 & 1,160 & 980 & 1,196 \\
\hline \multicolumn{7}{|l|}{ Non-Residents } \\
\hline Lower & & 0 & & 0 & & 0 \\
\hline Upper & & 17 & & 16 & & 16 \\
\hline Grad I & & 3 & & 3 & & 3 \\
\hline Grad II & & 0 & & 0 & & 0 \\
\hline Total & & 20 & & 19 & & 19 \\
\hline \multicolumn{7}{|l|}{ Total FTE } \\
\hline Lower & & 7 & & 32 & & 0 \\
\hline Upper & & 922 & & 982 & & 1,080 \\
\hline Grad I & & 184 & & 164 & & 135 \\
\hline Grad II & & 2 & & 0 & & 0 \\
\hline $\begin{array}{c}\text { Total FTE } \\
\text { (FL Definition) }\end{array}$ & 1,002 & 1,114 & 980 & 1,179 & 980 & 1,215 \\
\hline $\begin{array}{c}\text { Total FTE } \\
\text { (US Definition) }\end{array}$ & 1,338 & 1,484 & 1,338 & 1,574 & 1,338 & 1,620 \\
\hline \multicolumn{7}{|c|}{ Student Headcount in Medical Doctorate (Medicine, Dentistry, Veterinary) Programs } \\
\hline & \multicolumn{2}{|c|}{ 2008-09 } & \multicolumn{2}{|c|}{ 2009-10 } & \multicolumn{2}{|c|}{$2010-11$} \\
\hline & Funded & Actual & Funded & Actual & Funded & Estimated \\
\hline Florida Residents & \multirow{3}{*}{\multicolumn{6}{|c|}{$\mathrm{N} / \mathrm{A}$}} \\
\hline Non-Residents & & & & & & \\
\hline Total & & & & & & \\
\hline \multicolumn{7}{|c|}{$\begin{array}{l}\text { Notes: Florida definitions of FTE (Undergraduate FTE }=40 \text { and Graduate FTE }=32 \text { credit hours per FTE) } \\
\text { are used for all items except the row named Total FTE (US Definition), which is based on } \\
\text { an Undergraduate FTE }=30 \text { and Graduate FTE }=24 \text { credit hours. Actual Medical headcounts are based on } \\
\text { Fall enrollment data. }\end{array}$} \\
\hline
\end{tabular}


STATE UNIVERSITY SYSTEM - 2010 ANNUAL REPORT

University of South Florida--Sarasota-Manatee

\section{Table 3B. Enrollment by Location}

For each distinct location (main, branch, site, regional campus) with> 150 FTE.

SITE: USF Sarasota/Manatee

\begin{tabular}{|c|c|c|c|}
\hline FTE by LEVEL & $\begin{array}{c}\mathbf{2 0 0 8 - 0 9} \\
\text { Actual }\end{array}$ & $\begin{array}{c}\mathbf{2 0 0 9 - 1 0} \\
\text { Actual }\end{array}$ & $\begin{array}{c}\mathbf{2 0 1 0 - 1 1} \\
\text { Estimated }\end{array}$ \\
\hline Lower & 7 & 32 & 0 \\
\hline Upper & 922 & 982 & 1,080 \\
\hline Grad I & 184 & 164 & 135 \\
\hline Grad II & 2 & 0 & 0 \\
\hline Total & $\mathbf{1 , 1 1 4}$ & $\mathbf{1 , 1 7 9}$ & $\mathbf{1 , 2 1 5}$ \\
\hline
\end{tabular}

*These figures match the enrollment plan submitted earlier this year. 
STATE UNIVERSITY SYSTEM - 2010 ANNUAL REPORT

Section 4 - Undergraduate Education

University of South Florida--Sarasota-Manatee

Table 4A. Baccalaureate Degree Program Changes in AY 2009-2010

\begin{tabular}{|c|c|c|c|c|c|}
\hline $\begin{array}{c}\text { Title of Program } \\
\text { (add more rows as needed) }\end{array}$ & $\begin{array}{c}\text { Six-digit } \\
\text { CIP Code }\end{array}$ & $\begin{array}{c}\text { Degree } \\
\text { Level }\end{array}$ & $\begin{array}{c}\text { Date of } \\
\text { UBOT } \\
\text { Action }\end{array}$ & $\begin{array}{c}\text { Starting } \\
\text { or Ending } \\
\text { Term }\end{array}$ & Comments \\
\hline
\end{tabular}

New Programs

\begin{tabular}{|l|l|l|l|l|l|}
\hline N/A & & & & & \\
\hline & & & & & \\
\hline
\end{tabular}

Terminated Programs

\begin{tabular}{|c|l|l|l|l|l|}
\hline N/A & & & & & \\
\hline & & & & & \\
\hline
\end{tabular}

Suspended Programs

\begin{tabular}{|l|l|l|l|l|l|}
\hline N/A & & & & & \\
\hline & & & & & \\
\hline
\end{tabular}

New Programs Considered by University But Not Approved 


\begin{tabular}{|c|c|c|c|c|c|}
\hline \multicolumn{6}{|c|}{$\begin{array}{l}\text { STATE UNIVERSITY SYSTEM - } 2010 \text { ANNUAL REPORT } \\
\text { Section } 4 \text { - Undergraduate Education }\end{array}$} \\
\hline \multicolumn{6}{|c|}{ University of South Florida--Sarasota-Manatee } \\
\hline \multicolumn{6}{|c|}{ Table 4B. First-Year Persistence Rates } \\
\hline Term of Entry & 2004 & 2005 & 2006 & 2007 & 2008 \\
\hline $\begin{array}{l}\text { Cohort Size } \\
\text { Full-Time FTIC }\end{array}$ & \multicolumn{5}{|c|}{$\begin{array}{l}\text { Parsing of retention/graduation data at the campus-level cannot be completed at } \\
\text { this time. }\end{array}$} \\
\hline \multicolumn{6}{|c|}{ From Same University } \\
\hline \% Still Enrolled & \multicolumn{5}{|c|}{$\begin{array}{l}\text { Parsing of retention/graduation data at the campus-level cannot be con } \\
\text { this time. }\end{array}$} \\
\hline \multicolumn{6}{|c|}{$\begin{array}{l}\text { Table 4C. Federal Definition - Undergraduate Progression and Graduation Rates } \\
\text { for Full-Time First-Time-in-College (FTIC) Students }\end{array}$} \\
\hline Term of Entry & Fall 2000 & Fall 2001 & Fall 2002 & Fall 2003 & Fall 2004 \\
\hline $\begin{array}{c}\text { Cohort Size } \\
\text { Full-Time FTIC }\end{array}$ & \multicolumn{5}{|c|}{$\begin{array}{l}\text { Parsing of retention/graduation data at the campus-level cannot be completed at } \\
\text { this time. }\end{array}$} \\
\hline \multicolumn{6}{|l|}{6 - Year Rates } \\
\hline \multicolumn{6}{|c|}{ From Same University } \\
\hline$\%$ Graduated & \multirow{3}{*}{\multicolumn{5}{|c|}{$\begin{array}{l}\text { Parsing of retention/graduation data at the campus-level cannot be completed at } \\
\text { this time. }\end{array}$}} \\
\hline \% Still Enrolled & & & & & \\
\hline Success Rate & & & & & \\
\hline \multicolumn{6}{|c|}{$\begin{array}{l}\text { Notes: (1) Cohorts are based on undergraduate students who enter the institution in the Fall term (or Summer } \\
\text { term and continue into the Fall term); (2) Success Rate measures the percentage of an intial cohort of students } \\
\text { who have either graduated or are still enrolled. }\end{array}$} \\
\hline
\end{tabular}




\section{STATE UNIVERSITY SYSTEM - 2010 ANNUAL REPORT \\ Section 4 - Undergraduate Education Data \\ University of South Florida--Sarasota-Manatee}

\section{Table 4D. SUS - Undergraduate Progression and Graduation Rates}

for First-Time-in-College (FTIC) Students

\begin{tabular}{|c|c|c|c|c|c|}
\hline Term of Entry & Fall 2000 & Fall 2001 & Fall 2002 & Fall 2003 & Fall 2004 \\
\hline $\begin{array}{l}\text { Cohort Size } \\
\text { Full- E Part-Time }\end{array}$ & \multicolumn{5}{|c|}{$\begin{array}{l}\text { Parsing of retention/graduation data at the campus-level cannot be completed at } \\
\text { this time. }\end{array}$} \\
\hline \multicolumn{6}{|l|}{4 - Year Rates } \\
\hline \multicolumn{6}{|c|}{ From Same University } \\
\hline$\%$ Graduated & \multirow{2}{*}{\multicolumn{5}{|c|}{$\begin{array}{c}\text { Parsing of retention/graduation data at the campus-level cannot be completed at } \\
\text { this time. }\end{array}$}} \\
\hline \% Still Enrolled & & & & & \\
\hline \multicolumn{6}{|c|}{ From Other SUS Institution } \\
\hline$\%$ Graduated & \multirow{2}{*}{\multicolumn{5}{|c|}{$\begin{array}{l}\text { Parsing of retention/graduation data at the campus-level cannot be completed at } \\
\text { this time. }\end{array}$}} \\
\hline \% Still Enrolled & & & & & \\
\hline \multicolumn{6}{|c|}{ From State University System } \\
\hline$\%$ Graduated & \multirow{3}{*}{\multicolumn{5}{|c|}{$\begin{array}{l}\text { Parsing of retention/graduation data at the campus-level cannot be completed at } \\
\text { this time. }\end{array}$}} \\
\hline \% Still Enrolled & & & & & \\
\hline Success Rate & & & & & \\
\hline \multicolumn{6}{|l|}{6 - Year Rates } \\
\hline \multicolumn{6}{|c|}{ From Same University } \\
\hline$\%$ Graduated & \multirow{2}{*}{\multicolumn{5}{|c|}{$\begin{array}{l}\text { Parsing of retention/graduation data at the campus-level cannot be completed at } \\
\text { this time. }\end{array}$}} \\
\hline \% Still Enrolled & & & & & \\
\hline \multicolumn{6}{|c|}{ From Other SUS Institution } \\
\hline$\%$ Graduated & \multirow{2}{*}{\multicolumn{5}{|c|}{$\begin{array}{l}\text { Parsing of retention/graduation data at the campus-level cannot be completed at } \\
\text { this time. }\end{array}$}} \\
\hline \% Still Enrolled & & & & & \\
\hline \multicolumn{6}{|c|}{ From State University System } \\
\hline$\%$ Graduated & \multirow{3}{*}{\multicolumn{5}{|c|}{$\begin{array}{l}\text { Parsing of retention/graduation data at the campus-level cannot be completed at } \\
\text { this time. }\end{array}$}} \\
\hline \% Still Enrolled & & & & & \\
\hline Success Rate & & & & & \\
\hline \multicolumn{6}{|c|}{$\begin{array}{l}\text { Notes: (1) Cohorts are based on undergraduate students who enter the institution in the Fall term (or Summer } \\
\text { term and continue into the Fall term); (2) Success Rate measures the percentage of an intial cohort of students } \\
\text { who have either graduated or are still enrolled. }\end{array}$} \\
\hline
\end{tabular}




\begin{tabular}{|c|c|c|c|c|c|}
\hline \multicolumn{6}{|c|}{$\begin{array}{l}\text { STATE UNIVERSITY SYSTEM - } 2010 \text { ANNUAL REPORT } \\
\text { Section } 4 \text { - Undergraduate Education Data }\end{array}$} \\
\hline \multicolumn{6}{|c|}{ University of South Florida--Sarasota-Manatee } \\
\hline \multicolumn{6}{|c|}{$\begin{array}{l}\text { Table 4E. SUS - Undergraduate Progression and Graduation Rates } \\
\text { for AA Transfer Students }\end{array}$} \\
\hline Term of Entry & Fall 2002 & Fall 2003 & Fall 2004 & Fall 2005 & Fall 2006 \\
\hline $\begin{array}{l}\text { Cohort Size } \\
\text { Full- \& Part-Time }\end{array}$ & \multicolumn{5}{|c|}{$\begin{array}{l}\text { Parsing of retention/graduation data at the campus-level cannot be completed at } \\
\text { this time. }\end{array}$} \\
\hline \multicolumn{6}{|l|}{2 - Year Rates } \\
\hline \multicolumn{6}{|c|}{ From Same University } \\
\hline$\%$ Graduated & \multirow{2}{*}{\multicolumn{5}{|c|}{$\begin{array}{l}\text { Parsing of retention/graduation data at the campus-level cannot be completed at } \\
\text { this time. }\end{array}$}} \\
\hline \% Still Enrolled & & & & & \\
\hline \multicolumn{6}{|c|}{ From Other SUS Institution } \\
\hline$\%$ Graduated & \multirow{2}{*}{\multicolumn{5}{|c|}{$\begin{array}{l}\text { Parsing of retention/graduation data at the campus-level cannot be completed at } \\
\text { this time. }\end{array}$}} \\
\hline \% Still Enrolled & & & & & \\
\hline \multicolumn{6}{|c|}{ From State University System } \\
\hline$\%$ Graduated & \multirow{3}{*}{\multicolumn{5}{|c|}{$\begin{array}{l}\text { Parsing of retention/graduation data at the campus-level cannot be completed at } \\
\text { this time. }\end{array}$}} \\
\hline \% Still Enrolled & & & & & \\
\hline Success Rate & & & & & \\
\hline \multicolumn{6}{|l|}{4 - Year Rates } \\
\hline \multicolumn{6}{|c|}{ From Same University } \\
\hline$\%$ Graduated & \multirow{2}{*}{\multicolumn{5}{|c|}{$\begin{array}{l}\text { Parsing of retention/graduation data at the campus-level cannot be completed at } \\
\text { this time. }\end{array}$}} \\
\hline \% Still Enrolled & & & & & \\
\hline \multicolumn{6}{|c|}{ From Other SUS Institution } \\
\hline$\%$ Graduated & \multirow{2}{*}{\multicolumn{5}{|c|}{$\begin{array}{l}\text { Parsing of retention/graduation data at the campus-level cannot be completed at } \\
\text { this time. }\end{array}$}} \\
\hline \% Still Enrolled & & & & & \\
\hline \multicolumn{6}{|c|}{ From State University System } \\
\hline$\%$ Graduated & \multirow{3}{*}{\multicolumn{5}{|c|}{$\begin{array}{l}\text { Parsing of retention/graduation data at the campus-level cannot be completed at } \\
\text { this time. }\end{array}$}} \\
\hline \% Still Enrolled & & & & & \\
\hline Success Rate & & & & & \\
\hline \multicolumn{6}{|c|}{$\begin{array}{l}\text { Notes: (1) Cohorts are based on undergraduate students who enter the institution in the Fall term (or Summer } \\
\text { term and continue into the Fall term); (2) Success Rate measures the percentage of an intial cohort of students } \\
\text { who have either graduated or are still enrolled. }\end{array}$} \\
\hline
\end{tabular}




\begin{tabular}{|c|c|c|c|c|c|}
\hline \multicolumn{6}{|c|}{$\begin{array}{l}\text { STATE UNIVERSITY SYSTEM - } 2010 \text { ANNUAL REPORT } \\
\text { Section } 4 \text { - Undergraduate Education Data }\end{array}$} \\
\hline \multicolumn{6}{|c|}{ University of South Florida--Sarasota-Manatee } \\
\hline \multicolumn{6}{|c|}{$\begin{array}{l}\text { Table 4F. SUS - Undergraduate Progression and Graduation Rates } \\
\text { for Other Transfer Students }\end{array}$} \\
\hline Term of Entry & Fall 2001 & Fall 2002 & Fall 2003 & Fall 2004 & Fall 2005 \\
\hline $\begin{array}{l}\text { Cohort Size } \\
\text { Full- \& Part-Time }\end{array}$ & \multicolumn{5}{|c|}{$\begin{array}{l}\text { Parsing of retention/graduation data at the campus-level cannot be completed at } \\
\text { this time. }\end{array}$} \\
\hline \multicolumn{6}{|l|}{5 - Year Rates } \\
\hline \multicolumn{6}{|l|}{ From Same University } \\
\hline$\%$ Graduated & \multirow{2}{*}{\multicolumn{5}{|c|}{$\begin{array}{l}\text { Parsing of retention/graduation data at the campus-level cannot be completed a } \\
\text { this time. }\end{array}$}} \\
\hline$\%$ Still Enrolled & & & & & \\
\hline \multicolumn{6}{|c|}{ From Other SUS Institution } \\
\hline$\%$ Graduated & \multirow{2}{*}{\multicolumn{5}{|c|}{$\begin{array}{l}\text { Parsing of retention/graduation data at the campus-level cannot be completed at } \\
\text { this time. }\end{array}$}} \\
\hline \% Still Enrolled & & & & & \\
\hline \multicolumn{6}{|c|}{ From State University System } \\
\hline$\%$ Graduated & \multirow{3}{*}{\multicolumn{5}{|c|}{$\begin{array}{l}\text { Parsing of retention/graduation data at the campus-level cannot be completed a } \\
\text { this time. }\end{array}$}} \\
\hline$\%$ Still Enrolled & & & & & \\
\hline Success Rate & & & & & \\
\hline \multicolumn{6}{|c|}{$\begin{array}{l}\text { Notes: (1) Cohorts are based on undergraduate students who enter the institution in the Fall term (or Summer } \\
\text { term and continue into the Fall term); (2) Success Rate measures the percentage of an intial cohort of students } \\
\text { who have either graduated or are still enrolled. }\end{array}$} \\
\hline \multicolumn{6}{|c|}{ Table 4G. Baccalaureate Degrees Awarded } \\
\hline & 2005-2006 & 2006-2007 & 2007-2008 & 2008-2009 & 2009-2010 \\
\hline Baccalaureate Degrees & 355 & 384 & 427 & 450 & 357 \\
\hline \multicolumn{6}{|c|}{ Table 4H. Baccalaureate Degrees Awarded in Areas of Strategic Emphasis } \\
\hline & 2005-2006 & 2006-2007 & 2007-2008 & 2008-2009 & 2009-2010 \\
\hline Education & 3 & 2 & 10 & 11 & 0 \\
\hline Health Professions & 1 & 6 & 18 & 16 & 4 \\
\hline $\begin{array}{l}\text { Science, Technology, } \\
\text { Engineering, and Math }\end{array}$ & 14 & 13 & 18 & 23 & 10 \\
\hline $\begin{array}{c}\text { Security and } \\
\text { Emergency Services }\end{array}$ & 30 & 27 & 23 & 28 & 37 \\
\hline Globalization & 2 & 7 & 0 & 0 & 0 \\
\hline $\begin{array}{l}\text { TOTAL: Areas of } \\
\text { Strategic Emphasis }\end{array}$ & 50 & 55 & 69 & 78 & 51 \\
\hline
\end{tabular}




\section{STATE UNIVERSITY SYSTEM - 2010 ANNUAL REPORT \\ Section 4 - Undergraduate Education Data \\ University of South Florida--Sarasota-Manatee}

\section{Table 4I. Baccalaureate Degrees Awarded to Underrepresented Groups}

\begin{tabular}{|c|c|c|c|c|c|}
\hline & 2005-2006 & 2006-2007 & 2007-2008 & $\begin{array}{c}2008-2009 \\
\text { BASELINE YEAR }\end{array}$ & 2009-2010 \\
\hline \multicolumn{6}{|c|}{ Non-Hispanic Black Students } \\
\hline $\begin{array}{c}\text { Number of } \\
\text { Baccalaureate Degrees }\end{array}$ & 19 & 32 & 22 & $\begin{array}{c}26 \\
\text { Increase* }\end{array}$ & 15 \\
\hline $\begin{array}{c}\text { Percentage of All } \\
\text { Baccalaureate Degrees }\end{array}$ & $5.5 \%$ & $8.8 \%$ & $5.5 \%$ & $\begin{array}{c}5.9 \% \\
\text { Increase }^{*}\end{array}$ & $4.3 \%$ \\
\hline \multicolumn{6}{|l|}{ Hispanic Students } \\
\hline $\begin{array}{c}\text { Number of } \\
\text { Baccalaureate Degrees }\end{array}$ & 25 & 18 & 37 & $\begin{array}{c}27 \\
\text { Increase* }^{*}\end{array}$ & 26 \\
\hline $\begin{array}{c}\text { Percentage of All } \\
\text { Baccalaureate Degrees }\end{array}$ & $7.2 \%$ & $5.0 \%$ & $9.3 \%$ & $\begin{array}{c}6.2 \% \\
\text { Increase }^{*}\end{array}$ & $7.5 \%$ \\
\hline \multicolumn{6}{|l|}{ PELL-Grant Recipients } \\
\hline $\begin{array}{c}\text { Number of } \\
\text { Baccalaureate Degrees* }\end{array}$ & 151 & 138 & 153 & $\begin{array}{c}158 \\
\text { Increase }^{*}\end{array}$ & 161 \\
\hline $\begin{array}{c}\text { Percentage of All } \\
\text { Baccalaureate Degrees }\end{array}$ & $43.6 \%$ & $37.9 \%$ & $38.3 \%$ & $\begin{array}{c}36.3 \% \\
\text { Increase }^{*}\end{array}$ & $45.7 \%$ \\
\hline \multicolumn{6}{|c|}{$\begin{array}{l}\text { Note: PELL-Grant recipients are defined as those students who have received a PELL-Grant Within } 6 \text { Years of } \\
\text { Graduation. This does not include degrees awarded to students whose race/ethnicity code is missing (or not reported) or } \\
\text { for students who are non-resident aliens. Note*: Directional goals for the 2012-13 year as reported in the } 2010 \text { University } \\
\text { Workplan. }\end{array}$} \\
\hline
\end{tabular}

*Due to methodology changes in this data metric, campus-level figures may not roll up to the system numbers.

\section{Table 4J. Baccalaureate Completion Without Excess Credit Hours}

\begin{tabular}{|c|c|c|c|c|c|}
\hline & 2005-2006 & 2006-2007 & 2007-2008 & 2008-2009 & 2009-2010 \\
\hline $\begin{array}{c}\text { \% of Total Baccalaureate } \\
\text { Degrees Awarded Within } \\
110 \% \text { of Hours Required } \\
\text { for Degree }\end{array}$ & $\mathrm{N} / \mathrm{A}$ & N/A & $61.8 \%$ & $67.1 \%$ & $66.1 \%$ \\
\hline & Fall 2005 & Fall 2006 & Fall 2007 & Fall 2008 & Fall 2009 \\
\hline $\begin{array}{c}\text { Number of } \\
\text { Course Sections }\end{array}$ & 169 & 179 & 193 & 143 & 135 \\
\hline $\begin{array}{c}\text { Fewer than } 30 \\
\text { Students }\end{array}$ & $85.2 \%$ & $86.0 \%$ & $88.6 \%$ & $81.8 \%$ & $86.7 \%$ \\
\hline $\begin{array}{c}30 \text { to } 49 \\
\text { Students }\end{array}$ & $13.0 \%$ & $13.4 \%$ & $10.9 \%$ & $14.7 \%$ & $11.1 \%$ \\
\hline $\begin{array}{c}50 \text { to } 99 \\
\text { Students }\end{array}$ & $1.8 \%$ & $0.0 \%$ & $0.0 \%$ & $2.8 \%$ & $1.5 \%$ \\
\hline $\begin{array}{c}100 \text { or More } \\
\text { Students }\end{array}$ & $0.0 \%$ & $0.6 \%$ & $0.5 \%$ & $0.7 \%$ & $0.7 \%$ \\
\hline
\end{tabular}




\section{STATE UNIVERSITY SYSTEM - 2010 ANNUAL REPORT}

Section 4 - Undergraduate Education Data

\section{University of South Florida--Sarasota-Manatee}

Table 4L. Faculty Teaching Undergraduates

\begin{tabular}{|c|c|c|c|c|c|}
\hline & $2005-2006$ & $2006-2007$ & $2007-2008$ & $2008-2009$ & $2009-2010$ \\
\hline Percentage of Credit Hours Taught by: & $57.0 \%$ & $56.5 \%$ & $60.8 \%$ & $58.4 \%$ & $60.5 \%$ \\
\hline Faculty & $42.5 \%$ & $41.3 \%$ & $38.0 \%$ & $38.7 \%$ & $38.1 \%$ \\
\hline Adjunct Faculty & $0.4 \%$ & $2.2 \%$ & $1.0 \%$ & $2.6 \%$ & $0.7 \%$ \\
\hline Oraduate Students & $0.0 \%$ & $0.0 \%$ & $0.2 \%$ & $0.3 \%$ & $0.7 \%$ \\
\hline
\end{tabular}

Note: The definition of faculty varies for Tables $4 \mathrm{~L}, 4 \mathrm{M}$ and $4 \mathrm{~N}$. For Faculty Teaching Undergraduates, the definition of faculty is based on pay plans 01,02 , and 22.

\section{Table 4M. Undergraduate Instructional Faculty Compensation}

\begin{tabular}{|c|c|c|c|c|c|}
\hline & Fall 2005 & Fall 2006 & Fall 2007 & Fall 2008 & Fall 2009 \\
\hline $\begin{array}{c}\text { Average Salary and } \\
\text { Benefits for Faculty Who } \\
\text { Teach at Least One } \\
\text { Undergraduate Course }\end{array}$ & $\$ 80,236$ & $\$ 81,592$ & $\$ 87,377$ & $\$ 86,817$ & $\$ 87,857$ \\
\hline
\end{tabular}

Note: The definition of faculty varies for Tables $4 \mathrm{~L}, 4 \mathrm{M}$ and $4 \mathrm{~N}$. For Undergraduate Instructional Faculty Compensation, the definition of faculty is based on pay plan 22.

\section{Table 4N. Student/Faculty Ratio}

\begin{tabular}{|l|c|c|c|c|c|}
\hline & $2005-2006$ & $2006-2007$ & $2007-2008$ & $2008-2009$ & $2009-2010$ \\
\hline Student-to-Faculty Ratio & 21 & 21 & 26 & 23 & 19 \\
\hline
\end{tabular}

Note: The definition of faculty varies for Tables $4 \mathrm{~L}, 4 \mathrm{M}$ and $4 \mathrm{~N}$. For Student/Faculty Ratio, the definition of faculty is consistent with Common Data Set reporting (which counts full-time equivalent instructional faculty as full-time faculty plus $1 / 3$ part-time faculty). 


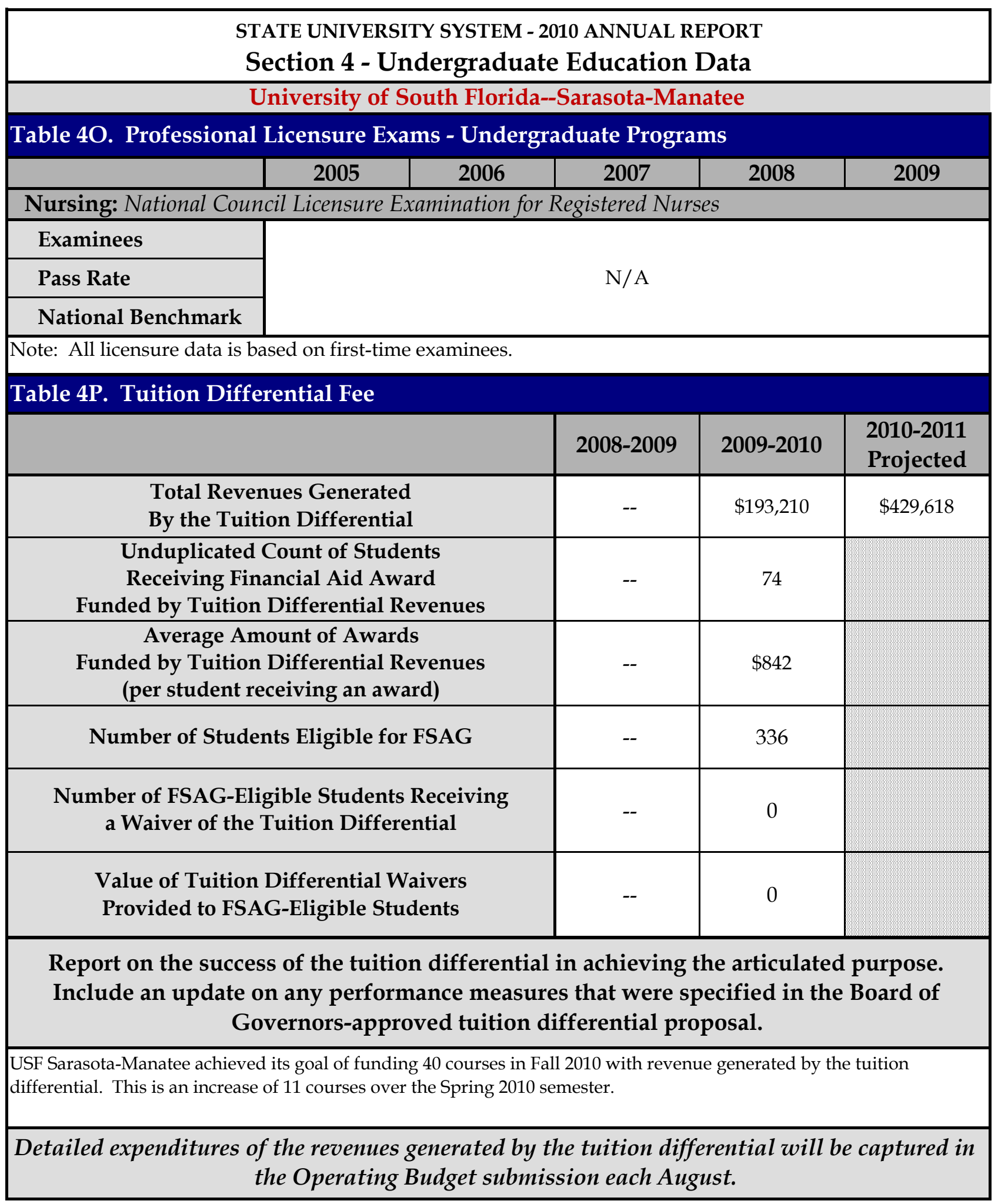




\section{STATE UNIVERSITY SYSTEM - 2010 ANNUAL REPORT}

Section 5 - Graduate Education Data

\section{University of South Florida--Sarasota-Manatee}

\section{Table 5A. GraduateDegree Programs Changes in AY 2009-2010}

Title of Program

(add more rows as needed)

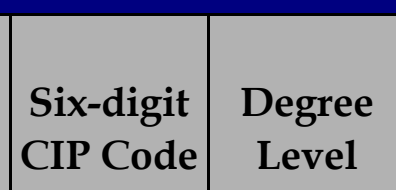

\begin{tabular}{c|c|c|c|c|} 
Date of & Starting \\
UBOT & or Ending \\
Action & Term
\end{tabular}

Date of

Board of Governors

\section{Comments}

New Programs

\begin{tabular}{|l|l|l|l|l|l|l|}
\hline N/A & & & & & & \\
\hline & & & & & & \\
\hline
\end{tabular}

Terminated Programs

N/A

(20.

\begin{tabular}{|l|l|l|l|l}
\hline & \\
\hline & &
\end{tabular}

\begin{tabular}{|l|l|l|l|l}
\hline & \\
\hline
\end{tabular}

\begin{tabular}{|l|l|l|l|l}
\hline & \\
\hline
\end{tabular}

Suspended Programs

\begin{tabular}{|l|l|l|l|l|l|l|}
\hline N/A & & & & & & \\
\hline & & & & & & \\
\hline
\end{tabular}

New Programs Considered by University But Not Approved 
STATE UNIVERSITY SYSTEM - 2010 ANNUAL REPORT

Section 5 - Graduate Education Data

University of South Florida--Sarasota-Manatee

Table 5B. Graduate Degrees Awarded

\begin{tabular}{|c|c|c|c|c|c|}
\hline & 2005-2006 & 2006-2007 & $2007-2008$ & 2008-2009 & 2009-2010 \\
\hline Master's and Specialist & 178 & 86 & 109 & 125 & 59 \\
\hline Research Doctoral & & & \multirow{6}{*}{$\mathrm{N} / \mathrm{A}$} & & \\
\hline Professional Doctoral & & & & & \\
\hline a) Medicine & & & & & \\
\hline b) Law & & & & & \\
\hline c) Pharmacy & & & & & \\
\hline $\begin{array}{l}\text { Research/ Professional } \\
\text { Doctoral, Combined }\end{array}$ & & & & & \\
\hline
\end{tabular}

Note: The total number of Professional Doctoral degrees includes other programs that are not specifically identified in lines $a, b$, and c.

Table 5C. Graduate Degrees Awarded in Areas of Strategic Emphasis

\begin{tabular}{|c|c|c|c|c|c|}
\hline & $2005-2006$ & $\mathbf{2 0 0 6 - 2 0 0 7}$ & $\mathbf{2 0 0 7 - 2 0 0 8}$ & $\mathbf{2 0 0 8 - 2 0 0 9}$ & $\mathbf{2 0 0 9 - 2 0 1 0}$ \\
\hline $\begin{array}{c}\text { Education } \\
\text { Critical Shortage Areas }\end{array}$ & 32 & 25 & 19 & 12 & 12 \\
\hline Health Professions & 3 & 2 & 9 & 7 & 4 \\
\hline $\begin{array}{c}\text { Science, Technology, } \\
\text { Engineering, and Math }\end{array}$ & 0 & 0 & 0 & 1 & 0 \\
\hline $\begin{array}{c}\text { Security and } \\
\text { Emergency Services }\end{array}$ & 20 & 0 & 13 & 3 & 4 \\
\hline Globalization & 0 & 0 & 0 & 0 & 0 \\
\hline TOTAL & 55 & $\mathbf{2 7}$ & $\mathbf{4 1}$ & $\mathbf{2 3}$ & $\mathbf{2 0}$ \\
\hline
\end{tabular}


STATE UNIVERSITY SYSTEM - 2010 ANNUAL REPORT

Section 5 - Graduate Education Data

University of South Florida--Sarasota-Manatee

Table 5D. Professional Licensure Exams - Graduate Programs

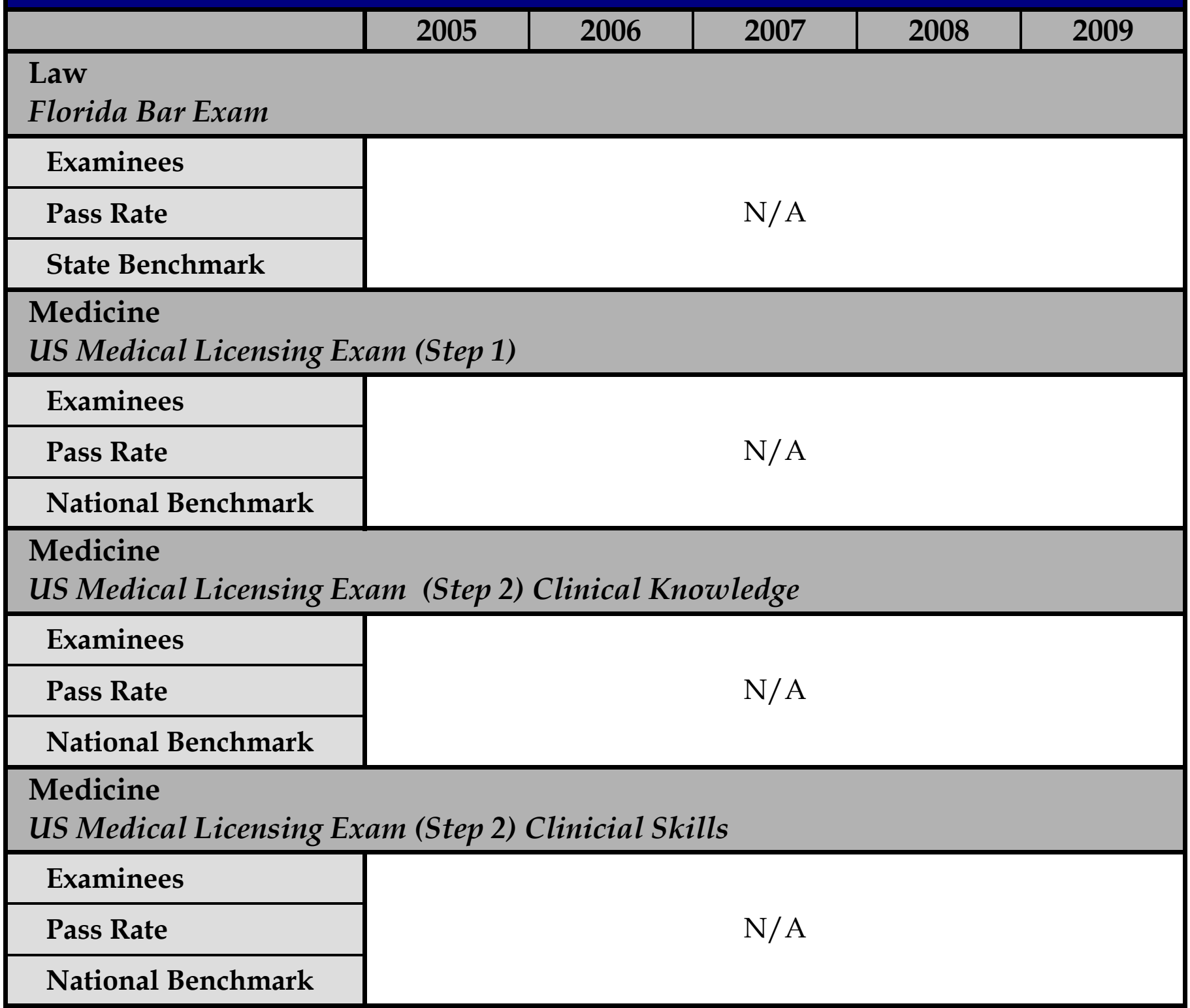

Note: All licensure data is based on first-time examinees. 
STATE UNIVERSITY SYSTEM - 2010 ANNUAL REPORT

Section 5 - Graduate Education Data

University of South Florida--Sarasota-Manatee

Table 5D. Professional Licensure Exams - Graduate Programs

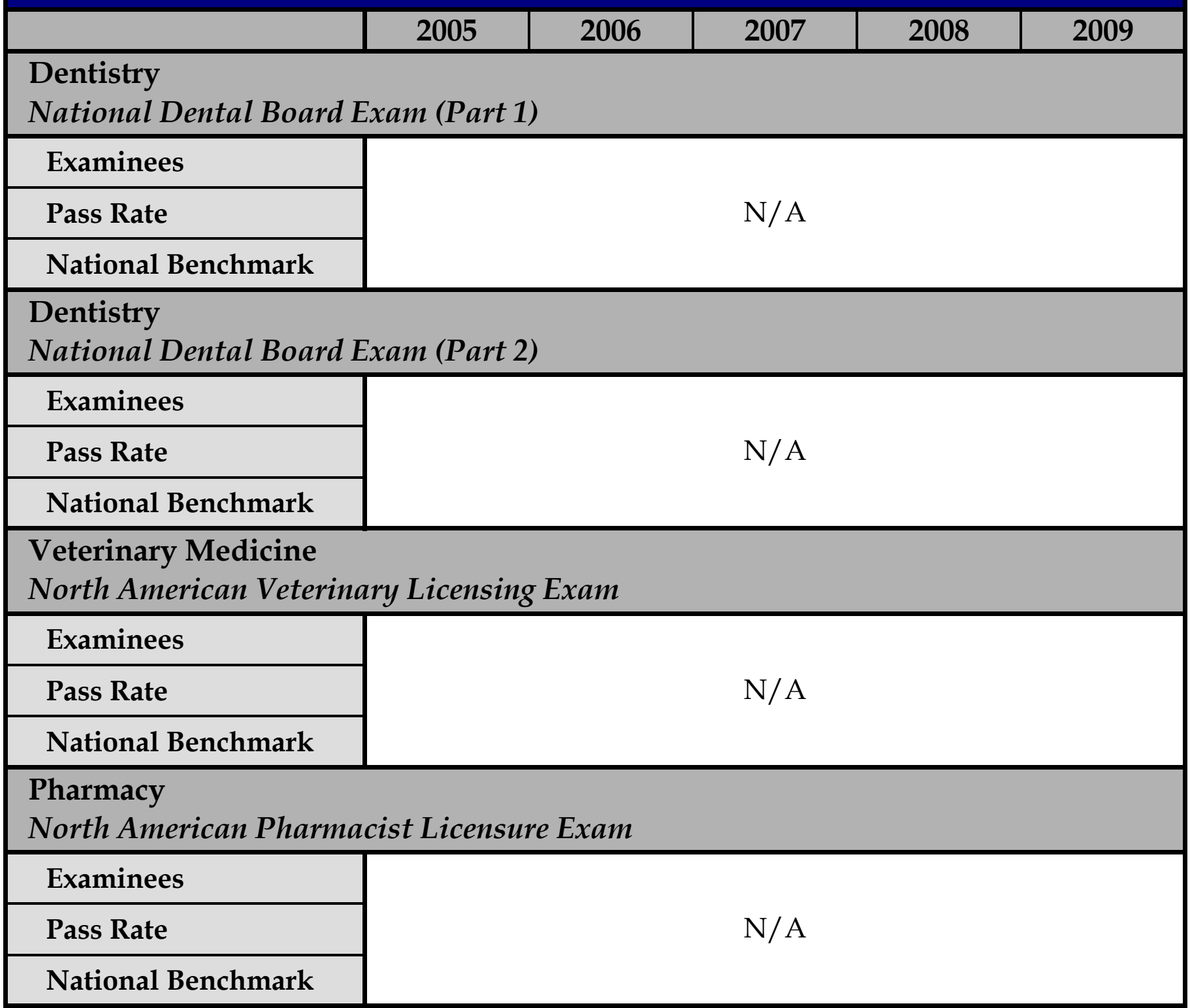

Note: All licensure data is based on first-time examinees. 


\begin{tabular}{|c|c|c|c|c|c|}
\hline \multicolumn{6}{|c|}{$\begin{array}{l}\text { STATE UNIVERSITY SYSTEM - } 2010 \text { ANNUAL REPORT } \\
\text { Section } 6 \text { - Research and Economic Development }\end{array}$} \\
\hline \multicolumn{6}{|c|}{ University of South Florida--Sarasota-Manatee } \\
\hline \multicolumn{6}{|c|}{ Table 6A. Research and Development } \\
\hline & 2004-2005 & 2005-2006 & 2006-2007 & 2007-2008 & 2008-2009 \\
\hline \multicolumn{6}{|c|}{ R\&D Awards (includes non-Science \& Engineering awards) } \\
\hline $\begin{array}{l}\text { Federally Funded Awards } \\
\text { (Thousand \$) }\end{array}$ & \multirow{2}{*}{\multicolumn{4}{|c|}{ Historical data not available at this time by campus. }} & $\$ 0$ \\
\hline $\begin{array}{l}\text { Total Awards } \\
\text { (Thousand \$) }\end{array}$ & & & & & $\$ 9$ \\
\hline \multicolumn{6}{|c|}{ R\&D Expenditures (includes non-Science \& Engineering expenditures) } \\
\hline $\begin{array}{l}\text { Federally Financed Expenditures } \\
\text { (Thousand \$) }\end{array}$ & \multirow{3}{*}{\multicolumn{5}{|c|}{$\begin{array}{l}\text { Development/Research data is a USF system-wide function. Consolidated system } \\
\text { data is only available at this time. }\end{array}$}} \\
\hline $\begin{array}{l}\text { Total Expenditures } \\
\text { (Thousand \$) }\end{array}$ & & & & & \\
\hline $\begin{array}{l}\text { Total R\&D Expenditures } \\
\text { Per Full-Time, Tenured, } \\
\text { Tenure-Earning Faculty Member } \\
\text { (\$) }\end{array}$ & & & & & \\
\hline \multicolumn{6}{|c|}{ Technology Transfer (as reported to AUTM) } \\
\hline Invention Disclosures & \multirow{6}{*}{\multicolumn{5}{|c|}{ DATA ARE ONLY APPLICABLE TO TAMPA CAMPUS }} \\
\hline Total U.S. Patents Issued & & & & & \\
\hline $\begin{array}{l}\text { Patents Issued Per 1,000 Full- } \\
\text { Time, Tenure and Tenure- } \\
\text { Earning Faculty }\end{array}$ & & & & & \\
\hline $\begin{array}{l}\text { Total Number of Licenses/ } \\
\text { Options Executed }\end{array}$ & & & & & \\
\hline $\begin{array}{l}\text { Total Licensing Income } \\
\text { Received (\$) }\end{array}$ & & & & & \\
\hline $\begin{array}{l}\text { Total Number of Start-Up } \\
\text { Companies }\end{array}$ & & & & & \\
\hline
\end{tabular}




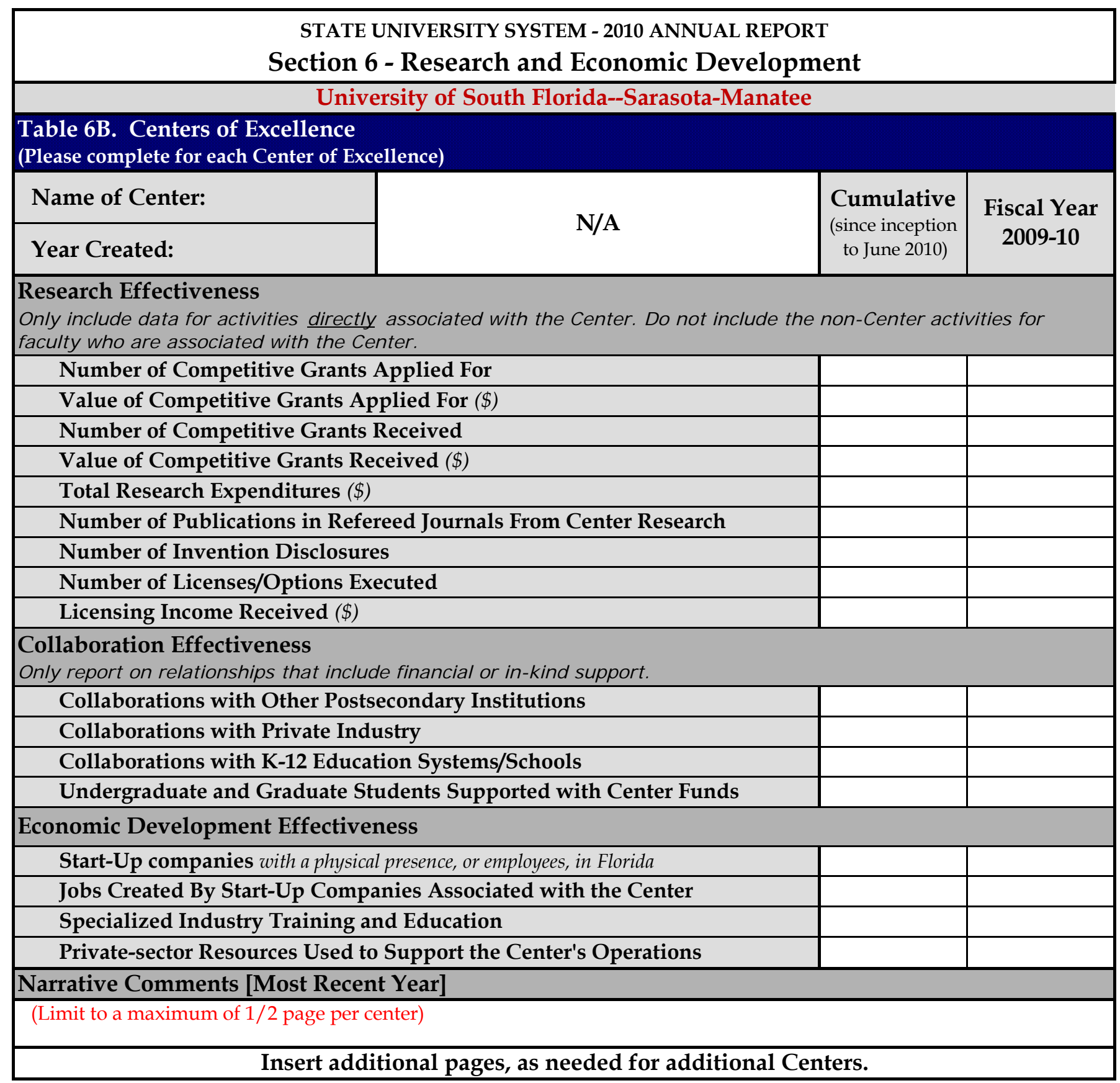




\begin{tabular}{|c|c|c|c|c|c|}
\hline \multicolumn{6}{|c|}{$\begin{array}{l}\text { STATE UNIVERSITY SYSTEM - } 2010 \text { ANNUAL REPORT } \\
\text { Section } 6 \text { - Research and Economic Development }\end{array}$} \\
\hline \multicolumn{6}{|c|}{ University of South Florida--Sarasota-Manatee } \\
\hline \multicolumn{6}{|c|}{ Table 6C. State University Research Commercialization Assistance Grants } \\
\hline \multirow{2}{*}{\multicolumn{4}{|c|}{ Project Name by Type of Grant }} & \multicolumn{2}{|c|}{ Cumulative } \\
\hline & & & & AWARDS & EXPENDITURES \\
\hline \multicolumn{4}{|l|}{ Phase I Grants } & & \\
\hline \multicolumn{4}{|l|}{ 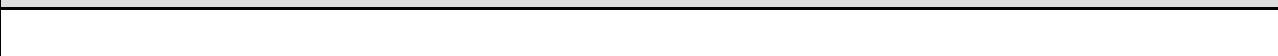 } & $\$ 0$ & $\$ 0$ \\
\hline \multicolumn{4}{|l|}{ Phase II Grants } & & \\
\hline \multicolumn{4}{|l|}{$\begin{array}{ll} \\
\end{array}$} & $\$ 0$ & $\$ 0$ \\
\hline \multirow{2}{*}{\multicolumn{4}{|c|}{ Phase III Grants }} & & \\
\hline & & & & $\$ 0$ & $\$ 0$ \\
\hline \multicolumn{4}{|l|}{ Total for all SURCAG Grants } & $\$ 0$ & $\$ 0$ \\
\hline \multicolumn{6}{|l|}{ Narrative Comments } \\
\hline \multirow{2}{*}{\multicolumn{6}{|c|}{$\begin{array}{l}\text { For each project, provide a brief update on: (1) the project's progress } \\
\text { and (2) the project's return on investment for the university and state } \\
\text { update on the project's ability to generate sufficient revenues to susta } \\
\text { Table 6D. 21st Century World Class Scholars Program }\end{array}$}} \\
\hline & & & & & \\
\hline & \multicolumn{2}{|c|}{ Grant Dollars } & \multicolumn{3}{|c|}{$\begin{array}{l}\text { Report the cumulative activity } \\
\text { since each scholar's award. }\end{array}$} \\
\hline $\begin{array}{l}\text { World Class Scholar(s) } \\
\text { and Field }\end{array}$ & $\begin{array}{c}\text { Amount } \\
\text { Awarded } \\
\text { (Thousand \$) }\end{array}$ & $\begin{array}{l}\text { Cumulative } \\
\text { Amount } \\
\text { Expended } \\
\text { (Thousand \$) }\end{array}$ & $\begin{array}{c}\text { External } \\
\text { Research } \\
\text { Awards } \\
\text { (Thousand \$) }\end{array}$ & $\begin{array}{l}\text { Patents } \\
\text { Filed / } \\
\text { Issued }\end{array}$ & $\begin{array}{c}\text { Licensing } \\
\text { Revenues } \\
\text { Generated } \\
(\$)\end{array}$ \\
\hline \multicolumn{6}{|c|}{ N/A } \\
\hline TOTAL for all Scholars & $\$ 0$ & $\$ 0$ & $\$ 0$ & 0 & $\$ 0$ \\
\hline Narrative Comments & & & & & \\
\hline
\end{tabular}

